\title{
The Structure of the $\mathrm{T}=147$ Iridovirus, CIV, at $13 \AA$ Resolution
}

X. Yan, *** P. R. Chipman,* A. J. Battisti, ${ }^{*}$ M. Bergoin, ${ }^{* * *}$ M. G. Rossmann, ${ }^{*}$ and T. S. Baker***

*Department of Biological Sciences, Purdue University, West Lafayette, IN 47907

**Departments of Chemistry\&Biochemistry and Molecular Biology, University of California, San

Diego, La Jolla, CA 92093-0378

***Lab of Comparative Pathology, University of MontpellierII, 34095 Montepellier cedex 5, France

Chilo iridescent virus (CIV) (genus Iridovirus, family Iridoviridae) infects the rice stem borer insect [1]. The CIV virion $\left(\sim 1 \times 10^{9} \mathrm{Da}\right)$ encapsidates a $209 \mathrm{kbp}$ dsDNA genome that is surrounded by a lipid membrane ( $\sim \%$ of total virion weight) [2]. More than 30 polypeptides can be differentiated with 2D gel electrophoresis [2]. The major capsid protein (MCP), P50 (467 amino acids, $51.4 \mathrm{kDa}$ ), and that of PBCV-1, Vp54, share $\sim 22 \%$ sequence identity [3-5]. The published 3D image reconstruction of CIV at $26 \AA$ resolution revealed that the protein shell (maximum diameter of $1850 \AA$ ) consists of 12 pentavalent (pentamer) and 1460 hexavalent (trimer) capsomers, arranged with $\mathrm{T}=147 \quad(h=7, k=7)$ icosahedral, quasi-equivalent symmetry [6]. The capsomers are organized into 12 pentasymmetrons and 20 trisymmetrons. Each pentasymmetron consists of one pentamer plus 30 trimers, whereas each trisymmetron consists of 55 trimers [6]. In this study, we have improved the resolution of the CIV image reconstruction to $13 \AA$.

Purified CIV samples were prepared for cryo-electron microscopy as described [6]. Images were recorded in an FEI/Philips CM300 FEG electron microscope at 33,000x nominal magnification with defocus levels ranging from 0.8 to $3.0 \mu \mathrm{m}$. A total of $6925 \mathrm{CIV}$ particle images were extracted from

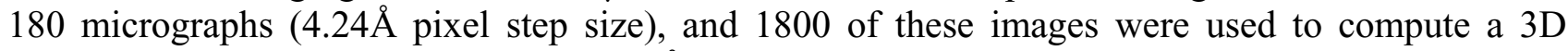
reconstruction to a resolution limit of $13 \AA$. Model-based procedures were used to determine and refine the particle origins and orientations with the previously published CIV reconstruction serving as the initial model $[6,7]$. The program SITUS was used to dock an atomic model of the PBCV-1 Vp54 trimer into each of the 25 trimers in the asymmetric unit of CIV [8].

The latest 3D image reconstruction of CIV reveals an enhanced view of the capsid shell, the protruding fibers, and the underlying bilayer membrane (Fig. 1A-D). Each trimeric capsomers exhibits a hexagonal profile and a central fiber that extends along the trimer axis. The fibers have both rigid (proximal part where density is highest) and flexible (distal part where density is weaker and smeared out) components. The bilayer membrane, which exhibits the characteristic railway track appearance, follows the general contour of the capsid shell. Some additional and slightly weaker densities occur between the capsid shell and the bilayer membrane. These densities may be responsible for tethering the lipid membrane to the outer capsid and thereby help maintain its icosahedral shape. The atomic model of Vp54 nicely fits within the reconstructed density of all CIV trimers. This result is consistent with the hypothesis that the MCPs of many large dsDNA viruses have closely similar tertiary structures despite only sharing weak sequence identity, and that these proteins may have evolved from a common ancestor [9].

\section{References}

[1] M. Fukaya et al., Appl. Entomol. Zool. 1 (1966) 69. 
[2] G. Devauchelle et al., Curr. Topics in Microbiol. and Immun. 116 (1985) 37.

[3] R. Stohwasser et al., J. Gen. Virol. 74 (1993) 873.

[4] M. Cerutti and G. Devauchelle. Virology 145 (1985) 123.

[5] N. Nandhagopal et al., Proc. Natl. Acad. Sci. 99 (2002) 14758.

[6] X. Yan et al., Nat. Struct. Biol. 7 (2000) 101.

[7] T. S. Baker and R. H. Cheng. J. Struct. Biology 116 (1996) 120.

[8] W. Wriggers et al., J. Struct. Biol. 125 (1999) 185.

[9] We thank R. Ashmore, Y. Ji and C. Xiao for assistance with programming. Work supported in part by grants from NIH (GM-33050) and NSF (MCR-9527131) to T.S.B. and from NIH (AI11219) to M.G.R., and a shared equipment grant from Keck Foundation to Purdue Structural Biology Group.

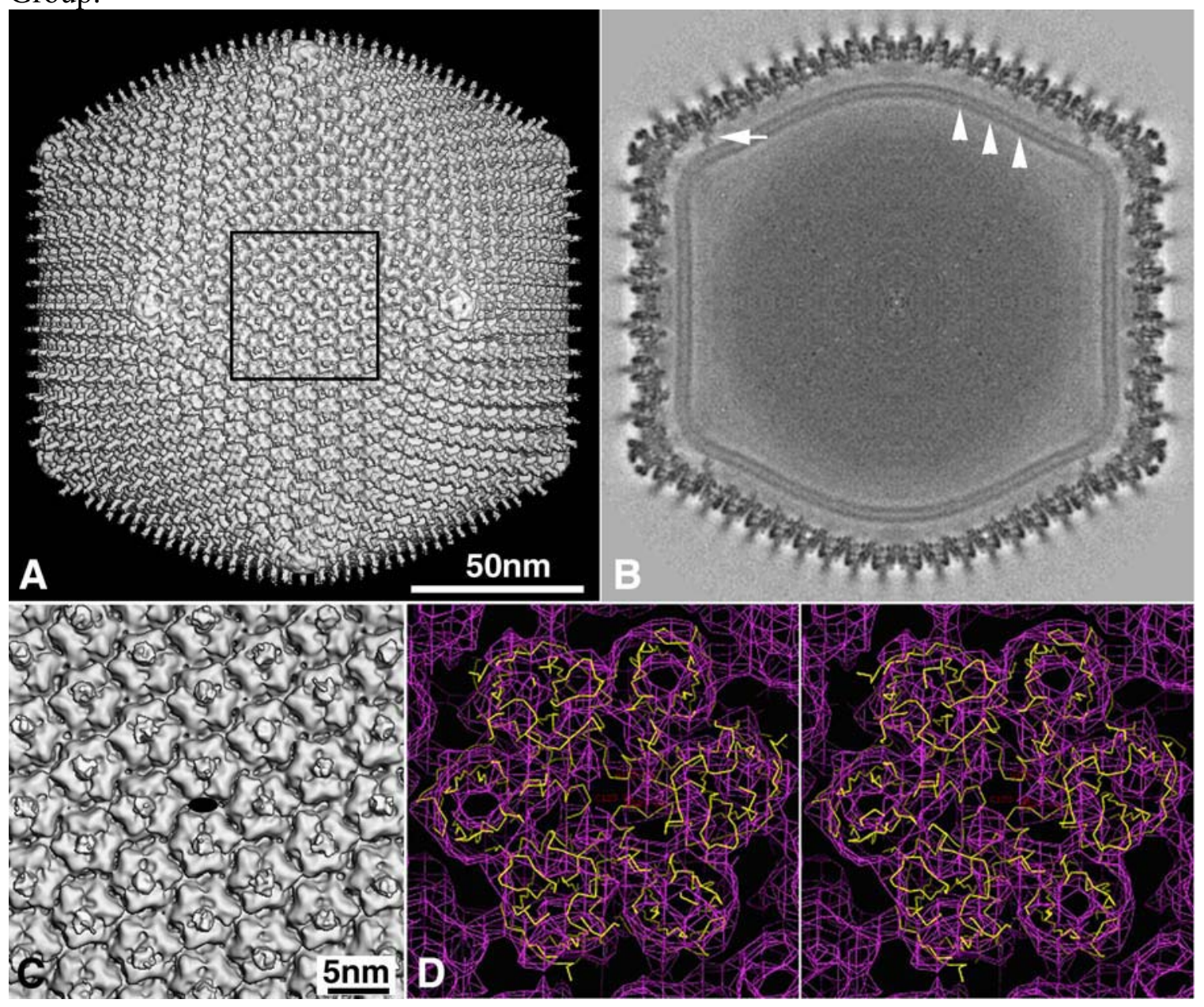

Fig.1. A. Shaded surface view of CIV 3D reconstruction at $13 \AA$ resolution, viewed along a 2-fold axis. B. Equatorial density section (high density appears darkest) view of CIV 3D map. The lipid bilayer ( $4.0 \AA$ thick, white arrowheads), which follows the inner contour of the capsid shell, appears to be tethered by numerous connections (e.g. white arrow) to the outer layer of capsomers. C. Closeup view of the region outlined by the square in (A). A black ellipse marks the location of a 2-fold axis. D. Stereo view (towards the center of the virus) showing the fit of an atomic model of the PBCV-1 major capsid protein, Vp54, into density for one CIV trimer. 